\title{
Trends in the use of adjuvant therapy for resected intrahepatic cholangiocarcinoma: getting ahead of the data
}

\author{
Jordan M. Cloyd, Timothy M. Pawlik \\ Department of Surgery, Division of Surgical Oncology, The Ohio State University Wexner Medical Center, Columbus, OH, USA \\ Correspondence to: Timothy M. Pawlik, MD, MPH, PhD. Professor and Chair, Department of Surgery, The Urban Meyer III and Shelley Meyer \\ Chair for Cancer Research, Professor of Surgery, Oncology, and Health Services Management and Policy, The Ohio State University, Wexner \\ Medical Center, 395 W. 12th Ave., Suite 670, Columbus, OH 43210, USA. Email: tim.pawlik@osumc.edu. \\ Comment on: Altman AM, Kizy S, Marmor S, et al. Adjuvant chemotherapy for intrahepatic cholangiocarcinoma: approaching clinical practice \\ consensus? Hepatobiliary Surg Nutr 2020;9:577-86.
}

Submitted May 06, 2021. Accepted for publication May 17, 2021.

doi: 10.21037/hbsn-2021-12

View this article at: http://dx.doi.org/10.21037/hbsn-2021-12

Intrahepatic cholangiocarcinoma (ICC), the second most common primary liver malignancy, is an aggressive cancer occurring with increasing global incidence. Significant advances in our understanding of its pathogenesis and molecular underpinnings, diagnostic and staging capabilities, and locoregional and systemic treatment options have occurred over the past several decades (1). Nevertheless, for patients with localized ICC, surgical management that includes margin negative resection and regional lymphadenectomy is the most important determinant of long-term outcomes. Unfortunately, even among patients with localized ICC who undergo prompt surgical resection by experienced multi-disciplinary teams, recurrence remain unacceptably high (2).

Given the high recurrence and overall poor outcomes experienced by the majority of patients with resected ICC, it is not surprising that clinicians and investigators have long been interested in identifying effective adjuvant therapies to treat micrometastatic disease, reduce the incidence of recurrence, and improve long-term survival outcomes. Indeed, the study by Altman et al. suggests that patients with resected ICC have been receiving adjuvant chemotherapy more often (3). In their retrospective review of the Surveillance Epidemiology and End Results (SEER) program database of patients with resected non-metastatic ICC, the authors reported that the use of adjuvant chemotherapy increased from $33 \%$ in $2000-2004$ to $41 \%$ in 2010-2014. These trends were even more significant among patients with metastatic lymph nodes (LN) (32\% to 60\%) and $\mathrm{T} 3 / \mathrm{T} 4$ disease (40\% to $60 \%)$. Interestingly, overall survival among all patients significantly increased during the same study period (median 32 to 41 months).

The increasing utilization of adjuvant chemotherapy for resected ICC is notable given the absence of strong efficacy data especially during the years studied by Altman et al. (2000-2014). While population-based datasets, cancer registries, and retrospective institutional data had supported the use of adjuvant chemotherapy and/or chemoradiation therapy in patients with resected ICC, particularly those with high-risk features such as $\mathrm{R} 1$ margin status or $\mathrm{LN}$ disease (4), randomized controlled trials have produced conflicting results. The PRODIGE 12-ACCORD 18 trial, initially presented in 2017 and formally published in 2019, randomized 196 patients (44\% ICC) with resected biliary tract cancers (BTCs) treated between 2010-2014; patients were randomized to either observation or adjuvant gemcitabine-oxaliplatin and noted no difference in recurrence-free or overall survival (5). Also published in 2019, the BILCAP trial randomized 447 patients with resected BTCs (19\% ICC) to either observation or adjuvant capecitabine and noted no difference in outcomes in the primary intention-to-treat analysis but improved overall survival among patients receiving adjuvant chemotherapy according to a per-protocol analysis (6). Of note, the BCAT, which randomized patients with resected BTCs (ICC excluded) to adjuvant gemcitabine versus observation, also did not demonstrate a difference in recurrence-free survival (7). Two other adjuvant therapy trials for resected 
BTCs are ongoing $(8,9)$.

The increasing adoption of adjuvant chemotherapy noted in the study by Altman et al. in the years 2000-2014 likely reflect clinician desire to do something to improve patient outcomes even if only based on low quality evidence. Similar trends have been observed in the increasing use of neoadjuvant therapy for high-risk localized ICC despite the lack of level I evidence to support its efficacy (10). The global interest to identify a suitable adjuvant therapy for resected BTCs, even if the quality of evidence is not overwhelming, may also be evident in the quick incorporation of adjuvant capecitabine into national treatment guidelines despite a non-significant primary endpoint in the BILCAP trial, as well as other published negative adjuvant trials (11). Based on recent events, it is likely that the observed trends toward increasing utilization of adjuvant chemotherapy will only continue to increase.

Clearly more research is needed to define the optimal patient selection and appropriate adjuvant therapies for patients with resected ICC. Hopefully, strong multidisciplinary interest in clarifying the role of adjuvant therapy will be used to inspire the design, enrollment, and completion of novel cooperative trials. ICC is a molecularly diverse cancer and frequent somatic mutations make it a disease susceptible to targeted therapies (12). Thus, clinical trials of personalized approaches to adjuvant therapy based on molecular profiling should be prioritized. Altman et al. identified LN metastasis as one of the strongest predictors of adjuvant chemotherapy use yet a near-majority of patients still did not undergo lymphadenectomy at the time of surgery-a disappointing trend consistent with other studies (13).

In conclusion, the use of adjuvant chemotherapy among patients with resected ICC has increased even prior to the reporting of randomized controlled trials and updated treatment guidelines. These data suggest a strong ongoing interest in refining multidisciplinary approaches for this aggressive cancer. As novel therapies and targeted approaches are being developed and studied in prospective clinical trials, continued emphasis of proven strategies, including performing a margin-negative resection with regional lymphadenectomy and multidisciplinary evaluation for adjuvant chemotherapy, will enable the best possible outcomes for patients with ICC.

\section{Acknowledgments}

Funding: None.

\section{Footnote}

Provenance and Peer Review: This article was commissioned by the editorial office, Hepatobiliary Surgery and Nutrition. The article did not undergo external peer review.

Conflicts of Interest: Both authors have completed the ICMJE uniform disclosure form (available at https://hbsn. amegroups.com/article/view/10.21037/hbsn-2021-12/coif). Dr. TMP serves as the unpaid editorial board member of Hepatobiliary Surgery and Nutrition. JMC has no conflicts of interest to declare.

Ethical Statement: The authors are accountable for all aspects of the work in ensuring that questions related to the accuracy or integrity of any part of the work are appropriately investigated and resolved.

Open Access Statement: This is an Open Access article distributed in accordance with the Creative Commons Attribution-NonCommercial-NoDerivs 4.0 International License (CC BY-NC-ND 4.0), which permits the noncommercial replication and distribution of the article with the strict proviso that no changes or edits are made and the original work is properly cited (including links to both the formal publication through the relevant DOI and the license). See: https://creativecommons.org/licenses/by-nc-nd/4.0/.

\section{References}

1. Ejaz A, Cloyd JM, Pawlik TM. Advances in the Diagnosis and Treatment of Patients with Intrahepatic Cholangiocarcinoma. Ann Surg Oncol 2020;27:552-60.

2. Cloyd JM, Ejaz A, Pawlik TM. The Landmark Series: Intrahepatic Cholangiocarcinoma. Ann Surg Oncol 2020;27:2859-65.

3. Altman AM, Kizy S, Marmor S, et al. Adjuvant chemotherapy for intrahepatic cholangiocarcinoma: approaching clinical practice consensus? Hepatobiliary Surg Nutr 2020;9:577-86.

4. Horgan AM, Amir E, Walter T, et al. Adjuvant therapy in the treatment of biliary tract cancer: a systematic review and meta-analysis. J Clin Oncol 2012;30:1934-40.

5. Edeline J, Benabdelghani M, Bertaut A, et al. Gemcitabine and Oxaliplatin Chemotherapy or Surveillance in Resected Biliary Tract Cancer (PRODIGE 12-ACCORD 18-UNICANCER GI): A Randomized Phase III Study. J Clin Oncol 2019;37:658-67.

6. Primrose JN, Fox RP, Palmer DH, et al. Capecitabine 
compared with observation in resected biliary tract cancer (BILCAP): a randomised, controlled, multicentre, phase 3 study. Lancet Oncol 2019;20:663-73.

7. Ebata T, Hirano S, Konishi M, et al. Randomized clinical trial of adjuvant gemcitabine chemotherapy versus observation in resected bile duct cancer. Br J Surg 2018;105:192-202.

8. Nakachi K, Konishi M, Ikeda M, et al. A randomized Phase III trial of adjuvant S-1 therapy vs. observation alone in resected biliary tract cancer: Japan Clinical Oncology Group Study (JCOG1202, ASCOT). Jpn J Clin Oncol 2018;48:392-5.

9. Stein A, Arnold D, Bridgewater J, et al. Adjuvant chemotherapy with gemcitabine and cisplatin compared to observation after curative intent resection of cholangiocarcinoma and muscle invasive gallbladder carcinoma (ACTICCA-1 trial) - a randomized,

Cite this article as: Cloyd JM, Pawlik TM. Trends in the use of adjuvant therapy for resected intrahepatic cholangiocarcinoma: getting ahead of the data. HepatoBiliary Surg Nutr 2021;10(4):515-517. doi: 10.21037/hbsn-2021-12 multidisciplinary, multinational phase III trial. BMC Cancer 2015;15:564.

10. Akateh C, Ejaz AM, Pawlik TM, et al. Neoadjuvant treatment strategies for intrahepatic cholangiocarcinoma. World J Hepatol 2020;12:693-708.

11. Shroff RT, Kennedy EB, Bachini M, et al. Adjuvant Therapy for Resected Biliary Tract Cancer: ASCO Clinical Practice Guideline. J Clin Oncol 2019;37:1015-27.

12. Banales JM, Marin JJG, Lamarca A, et al. Cholangiocarcinoma 2020: the next horizon in mechanisms and management. Nat Rev Gastroenterol Hepatol 2020;17:557-88.

13. Zhang XF, Chen Q, Kimbrough CW, et al. Lymphadenectomy for Intrahepatic Cholangiocarcinoma: Has Nodal Evaluation Been Increasingly Adopted by Surgeons over Time? A National Database Analysis. J Gastrointest Surg 2018;22:668-75. 